\title{
Determination of bone porosity based on histograms of 3D $\mu \mathrm{CT}$ images
}

\author{
M. Cieszko $\cdot$ Z. Szczepański $\cdot$ P. Gadzała
}

Received: 28 June 2014/Accepted: 7 October 2014/Published online: 17 October 2014

(c) The Author(s) 2014. This article is published with open access at Springerlink.com

\begin{abstract}
A new method is proposed for direct determination of bone porosity based on histograms of 3D $\mu \mathrm{CT}$ scans and for precise definition of the global image segmentation threshold, preserving assessed porosity in the reconstructed binary image of the bone sample. In this method, the normed histogram is considered to be a probability distribution of voxel density (CT number or gray level) in the scan. It is a linear combination of two distributions characterizing the frequency of occurrence of voxels of pore and matrix type with various densities. Volume porosity, in this model, defines the probability of pore voxel occurrence in the whole set of voxels in the scan of the sample. This parameter and the parameters of both probability distributions are determined by an optimization method. The new method was used to determine the porosity and segmentation thresholds for $\mu \mathrm{CT}$ images of two 3D samples of human cancellous bone. The results were compared with those determined by the standard method and Otsu's method. The new method allows the porosity and the image segmentation threshold to be determined even in cases where use of the other methods is questionable or impossible.
\end{abstract}

\section{Introduction}

Identification of the microscopic geometry of bone tissue and macroscopic parameters of its pore space structure is a very important issue in the study of the physical properties

M. Cieszko ( $\square) \cdot$ Z. Szczepański · P. Gadzała Institute of Mechanics and Applied Computer Science, Kazimierz Wielki University, Kopernika 1, 85-074 Bydgoszcz, Poland

e-mail: cieszko@ukw.edu.pl of such material. The internal bone structure determines its local mechanical properties and bone strength, as an element of the human skeleton, and also strongly influences processes that take place in the bone tissue.

There are many methods for identifying the microscopic pore geometry of porous materials and their macroscopic parameters, such as optical microscopy, ultrasonic microscopy and porosimetry, mercury porosimetry, electric spectroscopy, permeametry, and gas pycnometry. Microcomputed tomography $(\mu \mathrm{CT})[1-3]$ is another of these methods. It is a very modern, nondestructive method used in various branches of science and engineering [4-7] for identification of the spatial structure of heterogeneous materials and small physical objects. In this method, as in the computed tomography applied in medical diagnostics, $\mathrm{X}$-rays are used to achieve an image resolution of one micrometer.

Microtomographic images of samples of porous materials form a basis for the reconstruction of the microscopic pore space geometry or matrix architecture. This allows identification of the stochastic characteristics, microscopic and macroscopic parameters of the pore space and matrix structure, material constants, and their directional characteristics [8-18]. For this purpose, pure geometrical methods [9, 14, 19-21] and methods of simulation of physical processes at microscopic level $[12,13,16,18]$ are used.

The accuracy of the parameters and coefficients obtained in this way is directly determined by the reconstruction quality of the microscopic pore space geometry. It depends not only on the image resolution of the sample, but also on the quality of the image segmentation, i.e., on the quality of transformation of the microtomographic image with various gray levels to a binary image. The crucial step for this process is image thresholding, which defines a limiting value of the gray level that separates all points 
(voxels) of the scan into two subsets constituting the matrix and the pore space. One can distinguish six groups of image thresholding methods [22]: local [23, 24], global $[25,26]$, based on the shape of the histogram [27], and using such tools as clustering [15], entropy [28], and fuzzy logic.

Thresholding is also crucial in the standard methods of determining the porosity parameter from microtomographic images of the material samples. For a binarized image of porous material, its porosity is a simple measure of the voxel volume fraction representing pores in the sample.

The most popular methods of global thresholding based on the shape of the histogram include what will be called here the standard method [25], and Otsu's method [29]. These are often used in microtomographic image analysis of human and animal bones [2, 10, 25, 26, 30-32].

In the standard method, the image segmentation threshold is defined as the voxel density (CT number or gray level) for which the frequency of voxel occurrence in the sample of bone scan reaches a minimum between the two extremes of the histogram corresponding to the pore and matrix types of voxels. In the graph of the cumulative histogram, this threshold value defines the location of the inflection point in the vicinity of which changes in the pore and matrix volume fractions in the sample are the smallest. It also corresponds to the ultimate changes in the voxel density. The standard method of determining the segmentation threshold has been implemented in some computer microtomographs.

In Otsu's method of thresholding, applied to $\mu \mathrm{CT}$ images of bone samples, the histogram of the scan is divided into two parts by the unknown value of the binarization threshold. After normalization, they are used as probability distributions of the density of voxels of two types (pore and skeleton) defined on two separate ranges. This makes it possible to define expressions for the mean voxel density of both classes as functions of the binarization threshold, and to define the so-called between-class variance of the mean densities of voxels in the scan. This is a measure of the deviation of the mean densities of both classes from the mean density of all voxels in the scan. Maximization of the value of this variance is the criterion for determining the optimum value for the scan binarization threshold. This method is often used for automatic threshold selection for image segmentation, and is implemented in the numerical computing environment MATLAB.

The aim of this paper is to present a new method for determining the porosity parameter and the binarization threshold for $3 \mathrm{D} \mu \mathrm{CT}$ images of bone tissue in which the standard procedure for their assessment has been reversed. First, using the model-based approach, the bone porosity is determined directly from the histogram of the 3D $\mu \mathrm{CT}$ image. Next, the binarization threshold is calculated from a condition requiring the obtained porosity to be preserved in the reconstructed binary image of the bone sample.

In this paper, bone is considered as a macroscopically strongly inhomogeneous porous material with low porosity in regions of the cortical bone and with high porosity in regions of the cancellous bone. The spatial distribution of this parameter is a basic macroscopic characteristic of such a material, determining its mechanical properties, which are important in, for example, diagnostics of morbidities of the bone.

Due to the largely random nature of the origin of image blurring in $\mu \mathrm{CT}$ scans $[3,4,33,34]$, a stochastic mixture model of a scan of the bone sample is proposed here. In this model, all voxels in the scan are considered to be of pore or matrix type, the density of which is a random variable, and the normalized histogram of the scan represents the probability distribution of this variable. This distribution is assumed to be a linear combination of two distributions describing the frequency of occurrence of vowels with various densities in the sets of voxels of pore and matrix type. The porosity in the proposed model defines the frequency (probability) of occurrence of voxels of pore type in the whole set of voxels in the scan of the bone sample.

The porosity parameter and parameters of the density distributions of voxels of pore and matrix type are determined by an optimization method implemented in the numerical computing environment MATLAB, i.e., by matching the mathematical model of the histogram to the histogram of the scan of the bone sample. In the applied method, the porosity parameter is calculated independently from an expression that minimizes a particular function of the approximation error. The obtained porosity allows the binarization threshold of the $\mu \mathrm{CT}$ image to be immediately determined, since the porosity should be preserved in the reconstructed binary image.

The proposed method was used to determine the porosities and the threshold values of two cubic samples of cancellous bone with various porosities taken from different places on the $\mu \mathrm{CT}$ scan of a human condyle. To make the samples statistically representative, a size limit was established above which their histograms do not change considerably. The results were compared with those obtained by the standard method [25, 31] and Otsu's method [29]. It was shown that the porosity and threshold values of a sample with a small matrix fraction, as determined by the new method, are considerably smaller than those obtained using the standard method and Otsu's method. For porosity the differences are of a few percent, while for the segmentation threshold they are about $140 \%$ and $40 \%$, respectively. This strongly influences the quality of reconstruction of the microscopic geometry of the bone sample. 
Fig. 1 Microscopic

representations of the investigated bone samples: a tomogram of one crosssectional layer of human condyle; $\mathbf{b}$ tomogram of one layer of sample I

$(400 \times 400 \times 1$ voxels $) ;$ d tomogram of one layer of sample II $(400 \times 400 \times 1$ voxels); c, e histograms of samples I and II respectively. Internal graphs contain enlarged plots of the continuous parts of the histograms
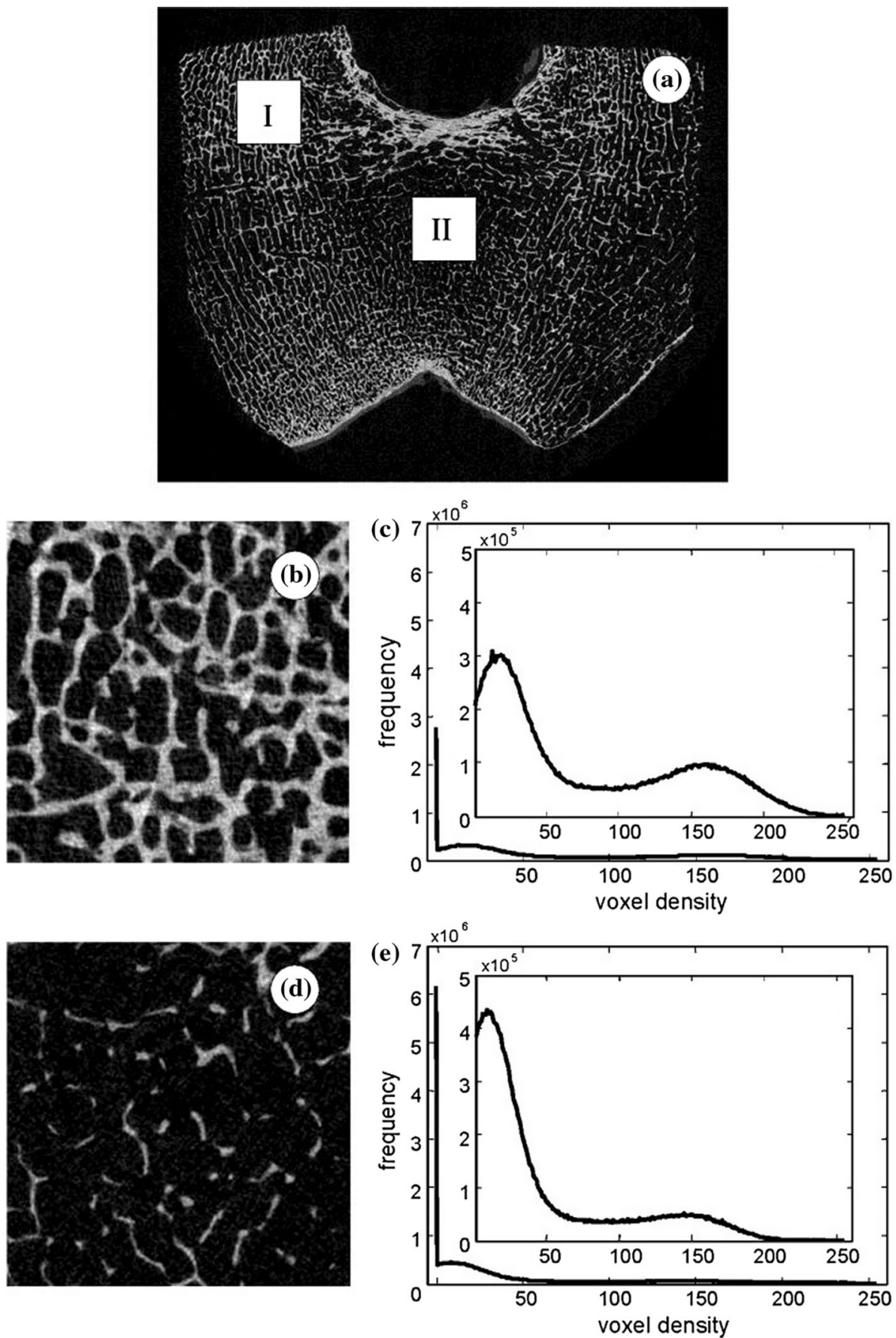

The proposed method may be used for determining the porosity and the binarization threshold of representative samples of various porous materials. Its multiple use also enables identification of the spatial distribution of both parameters in $\mu \mathrm{CT}$ images of inhomogeneous porous materials, and consequently allows more precise reconstruction of their microscopic structure.

\section{Characteristics of bone scan samples}

The new method of determining bone porosity based on a histogram of a 3D $\mu \mathrm{CT}$ image is presented using a scan of a human condyle performed on the microtomograph SkyScan 1172 with a voxel size of $17 \mu \mathrm{m}$. The tomogram of one cross-sectional layer of the investigated bone is shown in Fig. 1a. 
The gray levels in this figure represent the CT numbers of particular voxels in the layer of values from 0 to 255 , where 0 stands for black and represents voxels of pore type. For convenience, the CT number of voxel represented in the image by the voxel gray level will here be called the voxel density. The voxel density distribution in a scan corresponds to the mass density distribution in the scanned object, but does not represent this distribution directly.

We apply the methods of statistical analysis to investigate samples of the bone scan characterized locally by the density of voxels treated as a random variable. Due to the high macroscopic heterogeneity of bone, the voxel set in the whole bone scan cannot be considered as a study population, since all statistical characteristics of voxels have to be referenced to an area that can be recognized as homogeneous and representative in the statistical sense.

To make the analysis representative, two cubic samples of the scan of cancellous bone with sides of 400 voxels were taken from different places on the bone scan (Fig. 1a). One sample was taken from the lateral part of the bone scan (sample I) and the other from its central part (sample II). Enlarged images of one layer of both samples are shown in Fig. 1b and d. Their histograms are presented in Fig. 1c and e, respectively, and show the frequency of occurrence of voxels with the given density in the scans of the bone samples. After normalization, these curves can be interpreted as probability distributions of voxel density in the set of all voxels of the sample. Both histograms are discontinuous in the neighborhood of the point of zero density, and contain two visible extremes. In the range of lower values of the density, it corresponds to voxels of pore type, while in the range of higher values it corresponds to voxels of matrix type. The values of the histogram at the extreme points are different in both samples. This is caused by the larger volume fraction of pores in sample II in comparison with sample I, which is also visible in their tomograms.

The histograms of both samples of the bone scan also contain a considerable number of voxels with middle density values that cannot be uniquely attributed either to pores or to the matrix. This means that the choice of the threshold value of the density is very important for the segmentation process of samples of bone scans, and is crucial for the proper reconstruction of images of microscopic pore space geometry.

The dependence of the normalized histograms of both cubic bone samples on their size is shown in Fig. 2. For the sake of clarity, the discontinuous part of the histograms occurring in the neighborhood of the point of zero density is omitted. This figure shows that the histograms in both cases depend on the size of the sample, and the differences between them decrease as the size increases. For samples with side length greater than 100 voxels, the histograms are almost the same. Samples of the limiting size can be considered statistically representative for calculations of macroscopic parameters and material characteristics, the definitions of which are based on the volumetric relations in the sample, e.g., for porosity.

Taking into account that the binarization threshold of the sample scan is uniquely related to the sample porosity (see "Determination of the binarization threshold" section), the binarization threshold should, therefore, also be determined for representative samples. Otherwise, both parameters will be functions of the sample size and hence it will not be possible to consider them as macroscopic quantities. This means that the whole bone, as a macroscopically strongly inhomogeneous material, should be characterized by a function defining the spatial distribution of the porosity, and the high accuracy of reconstruction of its microscopic pore space structure based on $\mu \mathrm{CT}$ images requires determination of the spatial distribution of the scan binarization threshold.

The analysis presented here is performed using samples with a side length of 125 voxels.

\section{Model of the bone histogram}

Taking into account that the origins of the blurring of microtomographic images are of a random nature $[3,33$, 34], we derive a mathematical description of the histogram of the bone scan sample, taking a probabilistic mixture model of the histogram as a starting point. We assume that the quantized, three-dimensional sample of the scan of porous material constitutes a stochastic set of voxels with various densities (CT numbers) $\rho$ represented in the scan by gray levels. The set of voxels in the scan sample form the overall population of the analyzed voxels, and their density is a random variable, the probability distribution function of which, denoted by $\psi(\rho)$, we identify with the normalized histogram of the sample of porous material's scan. This defines the frequency of occurrence of voxels with the given density in the whole set of voxels composing the sample of the scan. We assume, however, that the set of voxels in the sample consists of two separate subsets (subpopulations): voxels of pore type and of matrix type. The frequencies of voxel occurrence in these subsets are described by the probability distributions $\psi_{\mathrm{p}}(\rho)$ and $\psi_{\mathrm{m}}(\rho)$, respectively. Both functions are defined on the whole domain of real numbers. This means that the attribution of a voxel of given density to the pore type or matrix type subset is of a stochastic nature, being described by the probability distributions $\psi_{\mathrm{p}}(\rho)$ and $\psi_{\mathrm{m}}(\rho)$.

To derive the relationship between the voxel density distribution $\psi(\rho)$ in the scan sample and the distributions $\psi_{\mathrm{p}}(\rho)$ and $\psi_{\mathrm{m}}(\rho)$ in the subsets of voxels of pore and matrix type, we determine the probability of the event $D^{\rho}$ of occurrence in the scan sample of voxels with density in the 

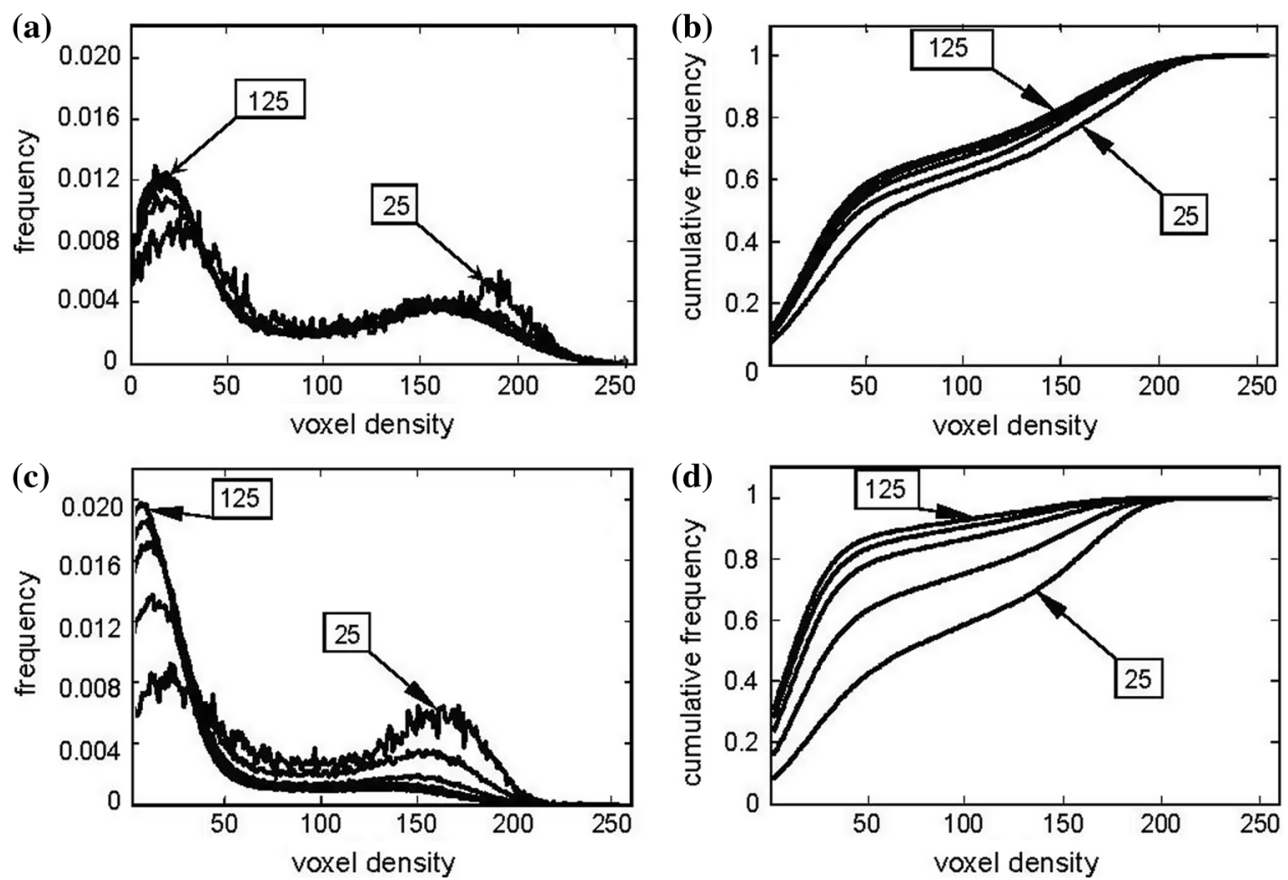

Fig. 2 Dependence of histograms $(\mathbf{a}, \mathbf{c})$ and cumulative histograms $(\mathbf{b}, \mathbf{d})$ on the size of cubic samples of bone scans (side lengths: $25,50 \ldots 125$ voxels): a, b sample I; c, $\mathbf{d}$ sample II

infinitesimal range $\langle\rho ; \rho+\mathrm{d} \rho\rangle$. We introduce the following notation:

$A$ - the event of occurrence of voxels of pore type in the scan sample.

$B$ - the event of occurrence of voxels of matrix type in the scan sample.

$\Omega$ - the set of the elementary events.

Events $A$ and $B$ are disjoint and their union forms the certain event,

$A \cap B=\Phi, A \cup B=\Omega$

where $\Phi$ denotes the empty set.

Therefore, the probabilities of these events can be represented in the form

$P(A)=f_{v}, P(B)=1-f_{v}$.

The parameter $f_{\mathrm{v}}$ defines the frequency (probability) of occurrence of voxels of pore type in the set of all voxels in the sample of the scan. Therefore, this parameter can be interpreted as a measure of fraction of the pore voxels in the sample. We assume that value of parameter $f_{\mathrm{v}}$ is equal to the volume fraction of pores (porosity) in the bone sample, the $\mu \mathrm{CT}$ image of which is analyzed.

Taking into account that $D^{\rho} \subset \Omega$, and applying the total probability theorem, we have

$P\left(D^{\rho}\right)=P\left(D^{\rho} \mid A\right) P(A)+P\left(D^{\rho} \mid B\right) P(B)$,

where $P\left(D^{\rho} \mid A\right)$ and $P\left(D^{\rho} \mid B\right)$ are conditional probabilities.

Since

$$
\begin{gathered}
\mathrm{P}\left(D^{\rho} \mid A\right)=\psi_{\mathrm{p}}(\rho) \mathrm{d} \rho, \mathrm{P}\left(D^{\rho} \mid B\right)=\psi_{\mathrm{m}}(\rho) \mathrm{d} \rho, \\
\mathrm{P}\left(D^{\rho}\right)=\psi(\rho) \mathrm{d} \rho,
\end{gathered}
$$

from (3.3) we obtain the relation

$\psi(\rho)=f_{v} \psi_{\mathrm{p}}(\rho)+\left(1-f_{v}\right) \psi_{\mathrm{m}}(\rho)$,

in which the porosity $f_{\mathrm{v}}$ of the sample of porous material is present explicitly.

Such probabilistic models are commonly applied for the statistical analysis of data in various fields of scientific research [35]. This includes clustering, handing missing data, modeling heterogeneity, density estimation, pattern recognition, and machine learning.

\section{Determination of porosity and other model parameters}

The probabilistic mixture model (3.5) of the histogram of a bone scan sample allows determination of complete information about the statistical characteristics and internal structure of the voxel set in the sample. This includes the porosity parameter $f_{\mathrm{v}}$ and the parameters describing the density distributions $\psi_{\mathrm{p}}(\rho)$ and $\psi_{\mathrm{m}}(\rho)$ of voxels in the pore and matrix type subsets. These parameters will be estimated here by an optimization method, fitting the mathematical model of the histogram to the histogram of the bone scan sample. Due to the rather free choice of the quantization limits of the scan, they cannot be identified with the limit values of the voxel densities in their 
distributions. Therefore, estimators of these parameters are determined during the optimization process.

Probability distribution functions

We assume that the probability distribution functions $\psi_{\mathrm{p}}(\rho)$ and $\psi_{\mathrm{m}}(\rho)$ occurring in the formula (3.5) have the form

$$
\begin{aligned}
\psi_{\kappa}(\rho) & =C_{\kappa} g_{\kappa}(\rho) \\
& =C_{\kappa} \frac{\left(\frac{\rho-a_{\kappa}}{b_{\kappa}-a_{\kappa}}\right)^{\alpha_{\kappa}-1}\left(\frac{b_{\kappa}-\rho}{b_{\kappa}-a_{\kappa}}\right)^{\beta_{\kappa}-1}}{\left(\frac{\rho-a_{\kappa}}{b_{\kappa}-a_{\kappa}}\right)^{\alpha_{\kappa}+\beta_{\kappa}}+\left(\frac{b_{\kappa}-\rho}{b_{\kappa}-a_{\kappa}}\right)^{\alpha_{\kappa}+\beta_{\kappa}}}(\kappa=\mathrm{p}, \mathrm{m}),
\end{aligned}
$$

where

$$
C_{\kappa}=\frac{\left(\alpha_{\kappa}+\beta_{\kappa}\right)}{\pi\left(b_{\kappa}-a_{\kappa}\right)} \sin \left(\pi \frac{\alpha_{\kappa}}{\alpha_{\kappa}+\beta_{\kappa}}\right)
$$

is the normalization coefficient.

This distribution can be obtained from the rational distribution of the form

$\eta(x)=\frac{\alpha+\beta}{\pi x_{o}} \sin \left(\frac{\alpha}{\alpha+\beta} \pi\right) \frac{\left(x / x_{o}\right)^{\alpha-1}}{1+\left(x / x_{o}\right)^{\alpha+\beta}}$

by conversion of the random variable $x$, defined on the infinite domain of the positive real numbers, to a variable $\rho$ given by the relation

$\frac{x}{x_{o}}=\frac{\rho-a}{b-\rho}$,

and defined on the interval $\langle a, b\rangle$. Due to the form of function (4.1) we will call it a modified beta distribution.

Distributions (4.1) are defined on the finite domain of voxel densities and can take a skew form. Their parameters have to satisfy the following conditions:

$b_{\kappa}>a_{\kappa}, \alpha_{\kappa}+\beta_{\kappa}>\alpha_{\kappa}>0 \quad(\kappa=\mathrm{p}, \mathrm{m})$.

In the case when

$\alpha_{\kappa} \geq 1, \quad \beta_{\kappa} \geq 1$,

the distributions take finite values over the whole range of voxel densities.

Taking into account that the distributions (4.1) contain four parameters for each type of voxels, the theoretical model of the histogram given by the formula (3.5) is a function of nine parameters. This provides the model with high flexibility.

\section{Optimization procedure}

The parameters of the mixture model can be estimated by various methods [35], e.g., moment matching, spectral, direct optimization, minimum message length, and maximum likelihood (as with the expectation maximization (EM) algorithm). These methods estimate the structure parameters of the analyzed overall population as well the distribution parameters of its sub-populations based on sampling of the overall population.

The investigated set of voxels in the sample of the bone scan, unlike typical objects of statistical research, form an overall population with a known value for the frequency of occurrence of each element (voxel density) in the population, with the exception of voxels with zero density ascribed to them during the quantization process. The distribution of voxel density in the scan sample, represented by the normed cumulative histogram, is composed using information about the densities of all voxels in the sample of the scan which form the overall population of the investigated set of voxels, and not only a statistical sample of them. This allows the use of direct optimization methods instead of statistical methods to estimate the model parameters of the histogram.

The optimization procedure applied here is based on the multi-parameter nonlinear regression method, implemented in the numerical computing environment MATLAB. In this method, the best fit of the theoretical model to the experimental data is obtained by minimization of the sum of squared residuals of the model, i.e., the differences between the values of the data and the fitted model.

Denoting the normed histogram of the sample of the bone scan by $h\left(\rho_{i}\right)$, the residuals $r\left(\rho_{i}\right)$ of the model take the form

$r\left(\rho_{i}\right)=h\left(\rho_{i}\right)-\psi\left(\rho_{i}\right)$,

where $\rho_{i}(i=1,2, \ldots N)$ is the voxel density in the bone scan sample.

Then, the objective function of the optimization problem can be defined in the form

$E\left(f_{v}, \boldsymbol{p}\right)=\sum_{i=1}^{N} r^{2}\left(\rho_{i}\right)=\sum_{i=1}^{N}\left(Y_{i}-f_{v} X_{i}\right)^{2}$,

where

$$
\begin{array}{r}
X_{i}=\psi_{\mathrm{p}}\left(\rho_{i}\right)-\psi_{\mathrm{m}}\left(\rho_{i}\right)=C_{\mathrm{p}} g_{\mathrm{p}}\left(\rho_{i}\right)-C_{\mathrm{m}} g_{\mathrm{m}}\left(\rho_{i}\right), \\
Y_{i}=h\left(\rho_{i}\right)-\psi_{\mathrm{m}}\left(\rho_{i}\right)=h\left(\rho_{i}\right)-C_{\mathrm{m}} g_{\mathrm{m}}\left(\rho_{i}\right),
\end{array}
$$

and

$\boldsymbol{p}=\left[a_{\mathrm{p}}, b_{\mathrm{p}}, \alpha_{\mathrm{p}}, \beta_{\mathrm{p}}, a_{\mathrm{m}}, b_{\mathrm{m}}, \alpha_{\mathrm{m}}, \beta_{\mathrm{m}}\right]$

represents the vector of parameters of distributions $\psi_{\mathrm{p}}(\rho)$ and $\psi_{\mathrm{m}}(\rho)$ given by relation (4.1).

Function (4.6) depends on nine parameters of the mixture model of the histogram: the porosity $f_{\mathrm{v}}$ and eight parameters of the distributions (4.1). Considering the normalization coefficients $C_{\mathrm{p}}$ and $C_{\mathrm{m}}$ as parameters, the distributions (4.1) become functions of five parameters that 
have to satisfy the normalization conditions (4.2). They play the role of constraints imposed on the model parameters. Such a change in the way of viewing the parameters of the objective function (4.6) is useful because it enables a reduction of the independent parameters that have to be determined directly in the optimization procedure.

Taking into account that the mixture model (3.5) of the histogram depends linearly on the porosity $f_{\mathrm{v}}$ and the normalization coefficients $C_{\mathrm{p}}$ and $C_{\mathrm{m}}$, their optimum values for given values of the remaining parameters can be determined effectively from the condition for the minimum of the objective function (4.6). The first derivatives of function (4.6) with respect to the parameters $f_{\mathrm{v}}, C_{\mathrm{p}}$, and $C_{\mathrm{m}}$ give the conditions

$$
\begin{aligned}
& \overline{X_{i} Y_{i}}-f_{v} \overline{X_{i}^{2}}=0, \quad \overline{Y_{i} \psi_{\mathrm{p}}^{i}}-f_{v} \overline{X_{i} \psi_{\mathrm{p}}^{i}}=0, \\
& \overline{Y_{i} \psi_{\mathrm{m}}^{i}}-f_{v} \overline{X_{i} \psi_{\mathrm{m}}^{i}}=0
\end{aligned}
$$

where

$$
\psi_{\kappa}^{i}=\psi_{\kappa}\left(\rho_{i}\right)=C_{\kappa} g_{\kappa}\left(\rho_{i}\right), \quad \overline{()_{i}}=\sum_{i=1}^{N}()_{i} / N .
$$

Due to relation (4.7) $)_{1}$ only two of the conditions in (4.8) are independent. Condition $(4.8)_{1}$ can be used to define the optimum value of the porosity $f_{\mathrm{v}}$; then the condition $(4.8)_{2}$, or equivalently $(4.8)_{3}$, defines the constraint imposed on the model parameters.

Applying condition (4.8) ${ }_{1}$ the objective function (4.6) reduces to the form

$E_{p}(\mathbf{p}) \equiv E\left(f_{v}, \mathbf{p}\right)=N\left(\overline{Y_{i}^{2}}-\left(\overline{X_{i} Y_{i}}\right)^{2} / \overline{X_{i}^{2}}\right)$.

The quantity $E_{p}(\mathbf{p})$ characterizes the error of the approximation, and its variance $\sigma^{2}$ is given by the formula

$\sigma^{2}=E_{p} / N=\overline{Y_{i}^{2}}-\left(\overline{X_{i} Y_{i}}\right)^{2} / \overline{X_{i}^{2}}$.

Since the number of data in the data set (in the sample of the bone scan) is very large, formally the law of large numbers and central limit theorem can be used to estimate the porosity distribution. Then expression $(4.8)_{1}$ defines the mean value of the porosity, and its variance $\sigma_{f_{v}}^{2}$ is given by

$\sigma_{f_{v}}^{2}=\sigma^{2} / \sum_{i=1}^{N} X_{i}^{2}=\left(\overline{Y_{i}^{2}} / \overline{X_{i}^{2}}-\left(\overline{X_{i} Y_{i}} / \overline{X_{i}^{2}}\right)^{2}\right) / N$.

This allows the confidence interval $f_{\mathrm{v}} \pm \Delta f_{\mathrm{v}}$ for the porosity to be constructed for the assumed confidence level.

In the optimization procedure applied here, the multiparameter nonlinear regression method is used repeatedly for each randomly chosen starting value of the model parameters. The procedure is stopped when the changes in the approximation error (4.9) become very small.

\section{Determination of the binarization threshold}

The purpose of the process of binarization of the scan of the sample of porous material is to produce a numerical representation of the sample's internal geometry which precisely reflects the geometry of the real object. This requires division of the whole set of voxels in the sample scan into two disjoint subsets representing the matrix and the pore space. Such a division is determined by the threshold value of voxel density, above which voxels are ascribed to the matrix, and below which they are ascribed to the pore space. In this case, the histogram of the sample scan with distributed voxel gray levels is transformed into a two-value histogram, and the sample image becomes binary. Therefore, the choice of the binarization threshold determines the quality of the reconstruction of the microscopic pore geometry and simultaneously defines the porosity of the sample.

The method proposed here for determining the porosity directly from the histogram of the sample scan, without prior reconstruction of its binary image, allows reversion of the order of determination of the porosity and the binarization threshold. This is possible because the binarization procedure should preserve the determined porosity of the sample. Therefore, the threshold of voxel density $\rho_{\mathrm{t}}$ should reach the value for which the probability of occurrence of voxels in the sample scan with densities smaller than this threshold (voxels ascribed to pores) is equal to the porosity. We obtain the condition

$\int_{a_{\mathrm{p}}}^{\rho_{\mathrm{t}}} \psi(\rho) d \rho=f_{v}$.

This means that the threshold density $\rho_{\mathrm{t}}$ and its confidence interval $\rho_{\mathrm{t}} \pm \Delta \rho_{\mathrm{t}}$ can be directly determined from the cumulative histogram when the value of the porosity $f_{\mathrm{v}}$ and its confidence interval $f_{\mathrm{v}} \pm \Delta f_{\mathrm{v}}$ are known. From condition (5.1) we have

$\Delta \rho_{\mathrm{t}}=\Delta f_{v} / \psi\left(\rho_{\mathrm{t}}\right)$.

Considering (3.6), the condition (5.1) can be written in the form

$\left(1-f_{\mathrm{v}}\right) \int_{a_{\mathrm{p}}}^{\rho_{\mathrm{t}}} \psi_{\mathrm{m}}(\rho) \mathrm{d} \rho=f_{\mathrm{v}} \int_{\rho_{\mathrm{t}}}^{b_{\mathrm{m}}} \psi_{\mathrm{p}}(\rho) \mathrm{d} \rho$,

which also allows another interpretation of this condition. The left side of equality (5.3) defines the probability of occurrence in the sample scan of voxels of matrix type with densities less than the threshold $\rho_{\mathrm{t}}\left(\rho<\rho_{\mathrm{t}}\right)$, whereas the right side of this condition defines the probability of occurrence of voxels of pore type with densities greater 
Table 1 Estimators of the histogram model parameters for bone sample scans I and II of human condyle

\begin{tabular}{|c|c|c|c|c|c|c|c|c|c|c|c|}
\hline \multicolumn{3}{|l|}{ Estimation } & \multicolumn{9}{|c|}{ Estimators of the histogram model parameters } \\
\hline Number & & $\sigma \times 10^{4}$ & $a_{\mathrm{p}}$ & $b_{\mathrm{p}}$ & $\alpha_{\mathrm{p}}$ & $\beta_{\mathrm{p}}$ & $a_{\mathrm{m}}$ & $b_{\mathrm{m}}$ & $\alpha_{\mathrm{m}}$ & $\beta_{\mathrm{m}}$ & $f_{\mathrm{v}}$ \\
\hline \multirow[t]{5}{*}{ Sample I } & I1 & 1.16 & -157 & 178 & 6.45 & 3.49 & 72 & 275 & 1.22 & 3.20 & 0.699 \\
\hline & $\mathrm{I} 2$ & 1.17 & -152 & 174 & 6.23 & 3.45 & 70 & 280 & 1.21 & 3.46 & 0.696 \\
\hline & I3 & 1.18 & -155 & 177 & 6.37 & 3.48 & 72 & 278 & 1.20 & 3.39 & 0.698 \\
\hline & I4 & 1.18 & -159 & 181 & 6.54 & 3.53 & 73 & 275 & 1.20 & 3.23 & 0.700 \\
\hline & I5 & 1.18 & -155 & 177 & 6.37 & 3.48 & 72 & 278 & 1.20 & 3.39 & 0.698 \\
\hline \multirow[t]{5}{*}{ Sample II } & II1 & 1.25 & -48 & 64 & 1.81 & 2.19 & 13 & 174 & 1.05 & 1.06 & 0.885 \\
\hline & II 2 & 1.27 & -49 & 65 & 1.85 & 2.25 & 12 & 179 & 1.07 & 1.11 & 0.885 \\
\hline & II 3 & 1.27 & -49 & 65 & 1.85 & 2.25 & 12 & 180 & 1.07 & 1.12 & 0.885 \\
\hline & II 4 & 1.27 & -49 & 65 & 1.86 & 2.25 & 12 & 182 & 1.07 & 1.15 & 0.885 \\
\hline & II5 & 1.27 & -49 & 65 & 1.85 & 2.24 & 12 & 180 & 1.07 & 1.12 & 0.885 \\
\hline
\end{tabular}

Fig. 3 Histograms (circles) of sample I (a) and II (b) and their approximations (solid lines) described by the mixture model for parameter estimators I-1 and II-1

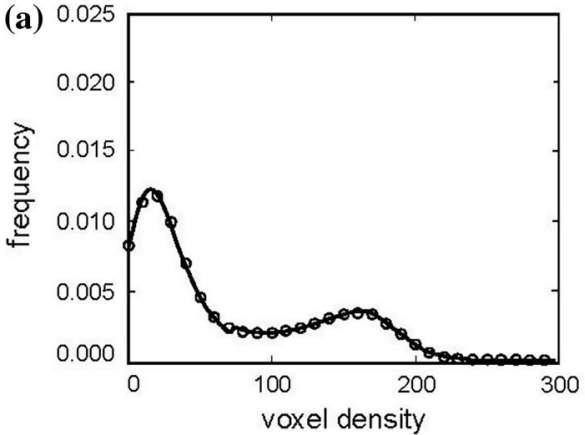

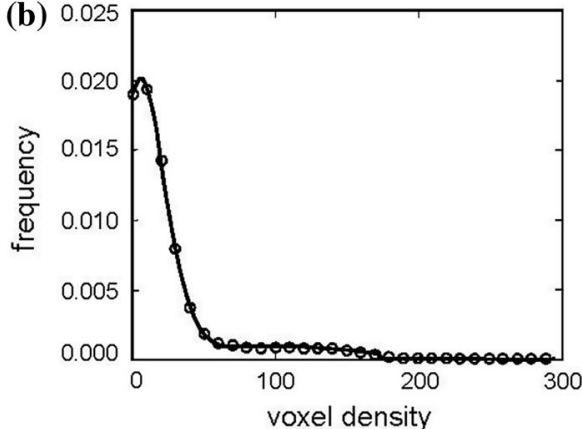

than this threshold $\left(\rho>\rho_{\mathrm{t}}\right)$. The equality of these expressions means that the number of voxels of matrix type ascribed to pores in the process of binarization has to be equal to the number of voxels of pore type ascribed to the matrix. This provides the internal compatibility of voxel division in the sample scan by the threshold $\rho_{\mathrm{t}}$ with the stochastic division of this set determined in the optimization process based on the histogram model.

\section{Results}

The values of the estimators of the histogram model parameters for the bone sample scans I and II, as determined by the optimization method described in subsection 4.2, are presented in Table 1. This gives the results of five example optimizations for each sample with the best fit of the mathematical model, obtained during the optimization process performed for different randomly chosen starting values of the model parameters. The table also gives the standard deviation $\sigma$ of the approximation error.

The graphs of the histograms of both samples and their example approximations described by the mixture model (3.5) and distributions (4.1) for the model parameter estimators I-1 and II-1 are shown in Fig. 3, and distributions of the approximation error $r(\rho)$ are presented in Fig. 4.
Table 2 gives the values of volume porosities and corresponding segmentation thresholds calculated from expressions (4.8) $)_{1}$ and (5.1), respectively, for the first three parameter estimators presented in Table 1 . The porosities and binarization thresholds are given together with their confidence intervals $\Delta f_{\mathrm{v}}$ and $\Delta \rho_{\mathrm{t}}$, calculated from the variance (4.11) and from relation (5.2), respectively, for the confidence level 0.99 .

For comparison, the estimators of the binarization threshold and the porosity parameter determined by the standard method and Otsu's method of image segmentation are also included in Table 2. Evaluation of the binarization threshold of sample II by the standard method is not clear, due to difficulties in establishing the position of the minimum of the histogram. In this work, the minima of both sample histograms are based on their local polynomial approximations.

Figure 5 illustrates how the segmentation threshold determined by the new and standard methods influences the quality of bone image reconstruction.

\section{Discussion}

The results of the optimization process described in the previous section show that the applied mixture model of 
Fig. 4 Distributions of the approximation error of the histograms of sample I (a) and II (b) from the mixture model for the parameter estimators I-1 and II-1
Table 2 Porosities and segmentation thresholds of samples I and II determined by the new and standard methods and by Otsu's method
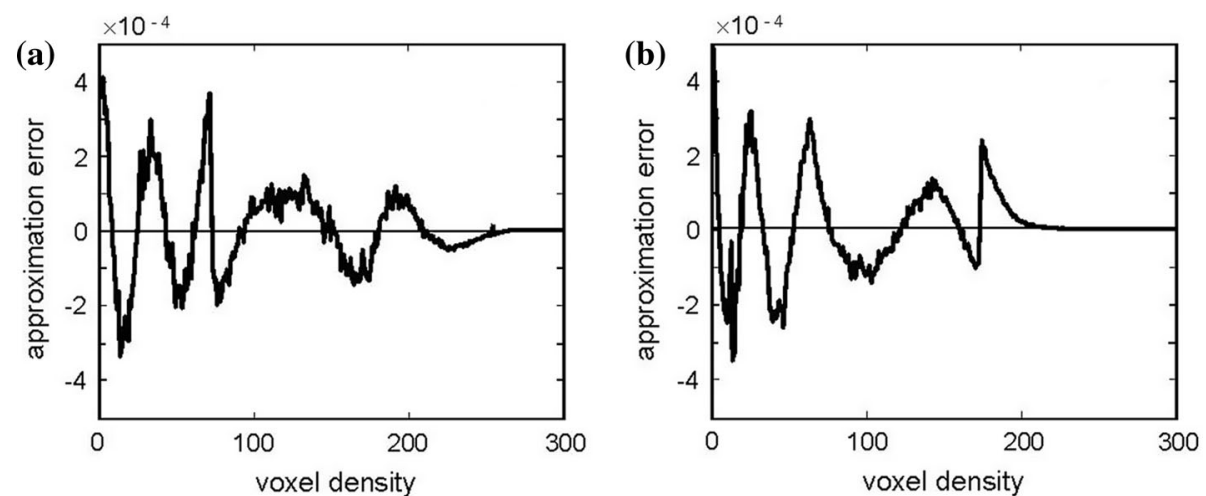

\begin{tabular}{|c|c|c|c|c|c|c|c|}
\hline \multicolumn{2}{|c|}{ Estimation number } & \multicolumn{2}{|l|}{ New method } & \multicolumn{2}{|c|}{ Standard method } & \multicolumn{2}{|c|}{ Otsu's method } \\
\hline & & $\overline{f_{\mathrm{v}} \pm \Delta f_{\mathrm{v}}}$ & $\rho_{\mathrm{t}} \pm \Delta \rho_{\mathrm{t}}$ & $\rho_{\mathrm{t}}^{\mathrm{s}}$ & $f_{\mathrm{v}}^{\mathrm{s}}$ & $\rho_{\mathrm{t}}^{\circ}$ & $f_{v}^{0}$ \\
\hline \multirow[t]{3}{*}{ Sample I } & I1 & $0.699 \pm 0.002$ & $89 \pm 1$ & 91 & 0.699 & 89 & 0.695 \\
\hline & I2 & $0.696 \pm 0.002$ & $88 \pm 1$ & & & & \\
\hline & I3 & $0.698 \pm 0.002$ & $89 \pm 1$ & & & & \\
\hline \multirow[t]{3}{*}{ Sample II } & II1 & $0.885 \pm 0.002$ & $47 \pm 1$ & 112 & 0.956 & 65 & 0.914 \\
\hline & II2 & $0.885 \pm 0.002$ & $47 \pm 1$ & & & & \\
\hline & III & $0.885 \pm 0.002$ & $47 \pm 1$ & & & & \\
\hline
\end{tabular}
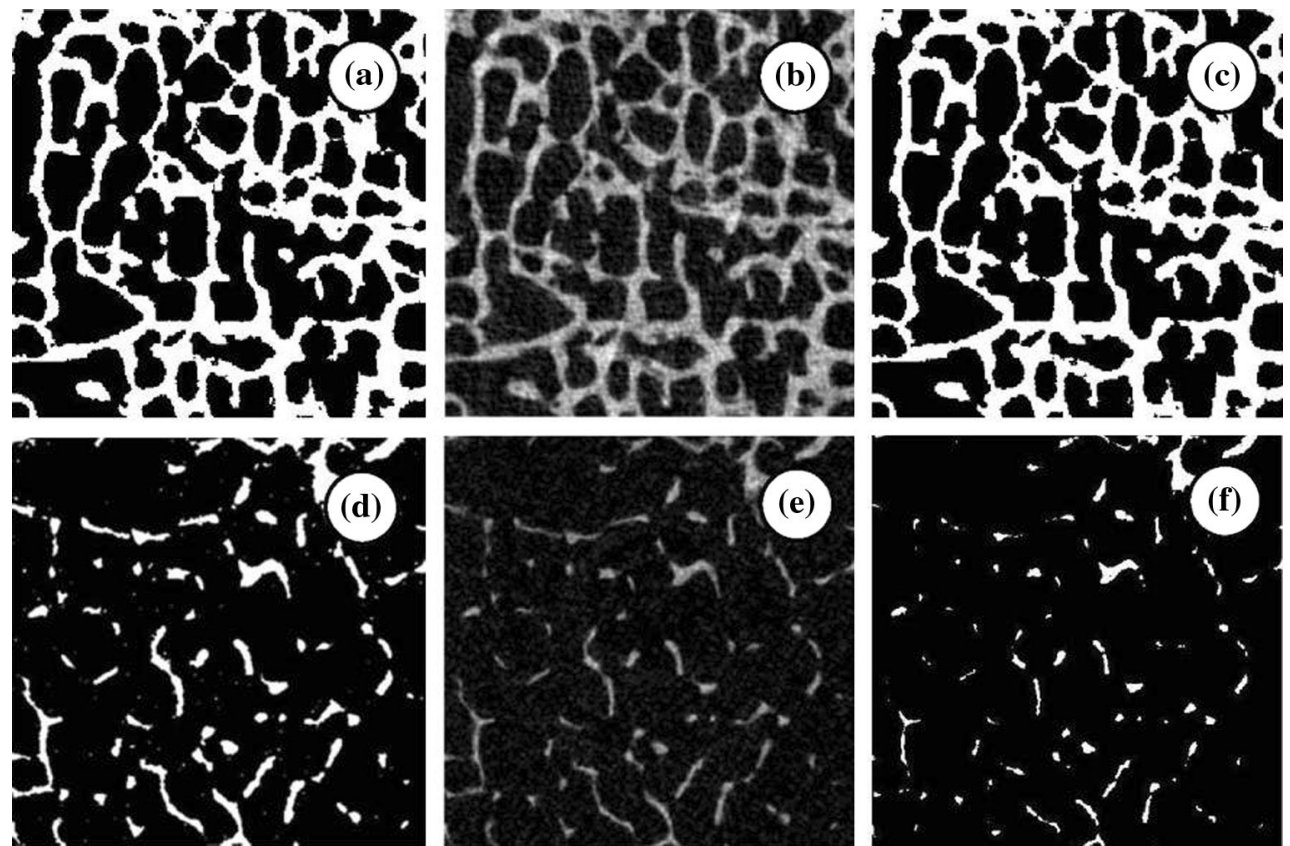

Fig. 5 Influence of the binarization thresholds determined by the new method (images: a) for $\rho_{\mathrm{t}}=88$ and $\mathbf{d}$ for $\rho_{\mathrm{t}}=43$ and the standard method (images: $\mathbf{c}$ for $\rho_{\mathrm{t}}=91$ and $\mathbf{f}$ for $\rho_{\mathrm{t}}=112$ ) on the quality of

the histogram and the modified beta distribution of the voxel density describe the histograms of both investigated samples of bone scans with high accuracy. This concerns image reconstruction. Tomogram $\mathbf{b}$ and its binary images $\mathbf{a}$ and $\mathbf{c}$ represent sample I, whereas tomogram $\mathbf{e}$ and its binary images $\mathbf{d}$ and f represent sample II

both qualitative (Fig. 3) and quantitative (Fig. 4) fitting of the theoretical and experimental curves. From Fig. 4 it results that the oscillations of the approximation error of 
both sample histograms are greater in the range of lower densities; however, their relative values are comparable in the whole range. The mean values of the approximation error of the histogram are: $\sigma=1.18 \times 10^{-4}$ and $\sigma=1.25 \times 10^{-4}$ for samples $\mathrm{I}$ and II, respectively (Table 1), and the estimators of the porosity parameter determined in consecutive realizations of the optimization procedure take values lying within very narrow intervals. For 20 optimizations performed for each sample, with starting parameters generated randomly, the estimators obtained for the porosity lie in the intervals $\langle 0.6940$, $0.7016\rangle$ and $\langle 0.8849,0.8853\rangle$ for samples I and II, respectively. This is the case in spite of some instability of the determined parameter estimators for the pore voxel density distribution, observed for sample I (Table 1). This is caused mainly by the lack of information about the form of the histogram in the part cut-off during the process of quantization of the scan.

The small values of the approximation error of the histograms determine very narrow confidence intervals for the porosity parameter estimator, even for a confidence level of 0.99 . They take the value $\Delta f_{v}=0.002$ for both samples (Table 2). This interval cannot, however, define the confidence interval of the porosity parameter of sample I determined by the optimization procedure, since in each realization of the optimization process the estimator of the porosity parameter takes different values outside this interval. In the case of sample II, the porosity parameter determined in the consecutive realizations of the optimization process belongs to the confidence interval.

A solution to this problem is to consider the procedure of determining the porosity as a random process that provides the assumed minimum level of fitting to the model histogram. Then the porosity becomes a random variable of this process, the stochastic characteristics of which can be determined based on numerical experimental data. For 20 realizations of the optimization procedure performed for sample I, the obtained mean value of the porosity parameter is $f_{v}^{\mathrm{I}}=0.698$, and its standard deviation and confidence interval for the confidence level 0.99 take the values $\sigma_{f_{v}}^{\mathrm{I}}=0.003, \Delta f_{v}^{\mathrm{I}}=0.007$. Finally, for both samples the following results were obtained:

$f_{v}^{\mathrm{I}}=0.698 \pm 0.007, f_{v}^{\mathrm{II}}=0.885 \pm 0.002$.

This allows the mean value of the scan binarization threshold $\rho_{t}$ and its confidence interval $\Delta \rho_{\mathrm{t}}$ to be determined. From relations (5.1) and (5.2) we have

$\rho_{t}^{\mathrm{I}}=88 \pm 3, \quad \rho_{t}^{\mathrm{II}}=47 \pm 1$.

The binarization threshold of sample II determined by the new method is much smaller than the thresholds determined by the standard method and Otsu's method
(Table 2). As a consequence, the porosities determined by these methods are also considerably different. These differences take values of about $138 \%$ and $38 \%$, respectively for the binarization threshold, and about $8 \%$ and $3 \%$ for the porosity. Nonetheless, the binarization threshold and the porosity of sample I determined by all three methods are almost the same. This substantial difference between the parameters of sample II determined by the new and standard methods is caused by the different levels of the models on which they are based. In the standard method, only information about one point of the histogram is used, whereas in the new method based on the stochastic model of the histogram, determination of image parameters is based on the information contained in the whole histogram. Therefore, the new method enables the porosity and the image segmentation threshold to be determined even in a case where application of the standard method is very difficult or impossible, i.e., when the histogram does not contain explicit extremes.

Detailed investigation of the relation between Otsu's method and the method proposed here is difficult on account of the different ways of using the histogram for determining the scan binarization threshold. Such a task would require detailed comparative analysis of both methods, which would be outside the scope of this work. Nevertheless, some qualitative evaluations can be formulated. The approach based on the mixture model of the histogram seems to be more fundamental. This method uses Eq. (5.1) for determining the scan binarization threshold, which can be interpreted as the primary definition of that threshold. Relation (5.1), written in the form (5.3), defines the statistical meaning of the thresholding process. Fulfillment of Eq. (5.3) provides the internal compatibility of the voxel division in the scan sample by the threshold density with the stochastic division of this set determined in the optimization process based on the histogram model. However, Otsu's method is formulated in a way that ensures that Eq. (5.1) is satisfied identically for any value of the scan binarization threshold. Instead of condition (5.1), an optimization procedure is proposed for determining the optimum value of the binarization threshold using the between-class variance of the voxel mean density in the scan sample as an objective function. The arbitrary choice of the objective function based on the mean densities of voxels of both classes ensures the simplicity of the optimization procedure, but it does not ensure its general nature.

The substantial difference between the binarization thresholds of sample II determined by the new method and the standard or Otsu's method is strongly influence the quality of reconstruction of its microscopic geometry. It is especially visible for the thresholds determined by the new and the standard methods, as shown in Fig. 5. 


\section{Conclusion}

A new method has been proposed here for determination of the bone porosity and image segmentation threshold based on the histogram of bone $\mu \mathrm{CT}$ scans. The novelty of this method consists in the use of a model-based approach that enables reversion of the procedure used in the other methods.

Use of the model-based approach and the optimization method for identification of the porosity and the image segmentation threshold enables consideration of all of the information contained in the histogram, and not only information regarding the position of its separated points or the mean values of voxel densities, as in the standard method or Otsu's method. This ensures the high accuracy of both determined parameters, and improves the quality of the image reconstruction of the microscopic pore space geometry. As a consequence, other macroscopic parameters, such as tortuosity and permeability, determined by simulation of physical processes in the pore space, can also be identified with better precision.

Moreover, the new method allows the porosity and the image segmentation threshold to be determined even in cases where application of the other methods is questionable or impossible.

Open Access This article is distributed under the terms of the Creative Commons Attribution License which permits any use, distribution, and reproduction in any medium, provided the original author(s) and the source are credited.

\section{References}

1. Feldkamp LA, Goldstein SA, Parfitt AM, Jesion G, Kleerekoper M (1989) The direct examination of three-dimensional bone architecture in vitro by computed tomography. J Bone Min Res $4: 3-11$

2. Rüegsegger P, Koller B, Muller R (1996) A microtomographic system for the nondestructive evaluation of bone architecture. Calcif Tissue Int 58:24-29

3. Davis GR, Wong F (1996) X-ray microtomography of bones and teeth. Physiol Meas 17:121-146

4. Stock SR (2008) Micro computed tomography: methodology and applications. CRC Press, Boca Raton

5. Mizutani R, Suzuki Y (2012) X-ray microtomography in biology. Review. Micron 43:104-115

6. Boller E, Cloetens P, Jl Baruchel et al (2006) Synchrotron X-ray microtomography: a high resolution, fast and quantitative tool for rock characterization. In: Desrues J, Viggiani G, Bésuelle P (eds) Advances in X-ray tomography for geomaterials, 7th edn. ISTE, London, pp 125-134. doi:10.1002/9780470612187

7. Tafforeau P, Boistel R, Boller E et al (2006) Applications of $\mathrm{X}$-ray synchrotron microtomography for non-destructive 3D studies of paleontological specimens. Appl Phys A83:195-202. doi:10.1007/s00339-006-3507-2

8. Von Rietbergen B, Odgaard A, Kabel J, Huiskes R (1998) Relationships between bone morphology and bone elastic properties can be accurately quantified using high-resolution computer reconstructions. J Orthop Res 16:23-28

9. Biswal B, Manwart C, Hilfer R (1998) Three-dimensional local porosity analysis of porous media. Phys A 255:221-241

10. Barbier A, Martel C, Vernejoul MC, Triode F, Nys M, Mocaer G, Morieux C, Murakami H, Lacheretz F (1999) The visualization and evaluation of bone architecture in the rat using threedimensional X-ray microcomputed tomography. J Bone Miner Metab 17:37-44

11. Lindquist WB, Venkatarangan A (1999) Investigating 3D geometry of porous media from high resolution images. Phys Chem Earth A 25:593-599

12. Ams CH, Knackstedt MA, Pinczewski MV, Martys MS (2004) Virtual permeametry on microtomographic images. J Pet Sci Eng 45:41-46

13. Ams CH, Knackstedt MA, Pinczewski MV, Garboczi EJ (2002) Computation of linear elastic properties from microtomographic images: methodology and agreement between theory and experiment. Geophysics 67:1396-1405

14. Torquato S (2002) Statistical description of microstructures. Ann Rev Mater 32:77-91

15. Taud H, Martinez-Angeles R, Parrot JF, Hernandez-Escobedo L (2005) Porosity estimation method by X-ray computed tomography. J Pet Sci Eng 47:209-217

16. Fedrich JT, Di Giovanni AA, Noble DR (2006) Predicting macroscopic transport properties using microscopic image data. J Geophys Res 111:B03201. doi:10.1029/2005JB00 3774

17. Sierpowska J, Hakulinnen MA, Toyras J, Day JS, Weinans H, Kiviranta I, Jurvelin JS, Lappalainnen R (2006) Interrelationships between electrical properties and microstructure of human trabecular bone. Phys Med Biol 51:5289-5303

18. Krabbenhaft K, Hain M, Wiggors P (2008) Computation of effective cement paste diffusivities from microtomographic images. Comp Methods Appl Sci 9:281-297

19. Torquato S (2002) Random heterogeneous materials: microstructure and macroscopic properties. Springer, New York

20. Hilfer R (1992) Local porosity theory flow in porous media. Phys Rev B 45:7115-7121

21. Hilfer R (1993) Local porosity theory for electrical and hydrodynamical transport through porous media. Phys A 194:406-414

22. Rajagopalan S, Yaszemski MJ, Robb R (2004) Evaluation of thresholding techniques for segmenting scaffold images in tissue engineering. Proc SPIE 5370:1456-1465

23. Waarsing JH, Day JS, Weinans H (2004) An improved segmentation method for in vivo $\mu \mathrm{CT}$ imaging. $\mathrm{J}$ Bone Miner Res 19:1640-1650

24. Müller R, Hildebrand T, Rüegsegger P (1994) Non-invasive bone biopsy: a new method to analyze and display the three-dimensional structure of trabecular bone. Phys Med Biol 39:145-164

25. Ding M, Odgaard A, Hvid I (1999) Accuracy of cancellous bone volume fraction measured by micro-CT scanning. J Biomech 32:323-326

26. Beaupied H, Chappard C, Basillais A, Lespessailles E, Benhamou CL (2006) Effect of specimen conditioning on the microarchitectural parameters of trabecular bone assessed by micro-computed tomography. Phys Med Biol 51:4621-4634

27. Rosenfeld A, Torre P (1983) Histogram concavity analysis as an aid in threshold selection. IEEE Trans Syst Man Cybern SMC 2:231-235

28. Kapur JN, Sahoo AKC, Wong A (1985) A new method for graylevel picture thresholding using the entropy of the histogram. Graph Model Image Process 29:273-285

29. Otsu N (1979) Threshold selection method from gray-level histograms. IEEE Trans Syst Men Cybern SMC 9:62-66 
30. Lima ICB, Oliveira LF, Lopes RT (2006) Bone architecture analyses of rat femur with 3D microtomographics images. J Radioanal Nucl Chem 269:639-642

31. Scanco Medical AG (1997) MicroCT 20 User's Guide, Software Revision 2.1:54-55

32. Palacio-Mancheno PE, Larriera AI, Doty SB, Cardoso L, Fritton SP (2014) 3D assessment of cortical bone porosity and tissue mineral density using high-resolution $\mu \mathrm{CT}$ : effects of resolution and threshold method. J Bone Miner Res 29:142-150
33. Van Geet M, Swennen R, Wevers M (2000) Quantitative analysis of reservoir rocks by microfocus X-ray computerized tomography. Sediment Geol 132:25-36

34. Ketcham RA, Carlson WD (2001) Acquisition optimization and interpretation of X-ray computed tomographic imagery: applications to geosciences. Comput Geosci 27:381-400

35. McLachlan G, Peal D (2000) Finite mixture models. Wiley, New York 